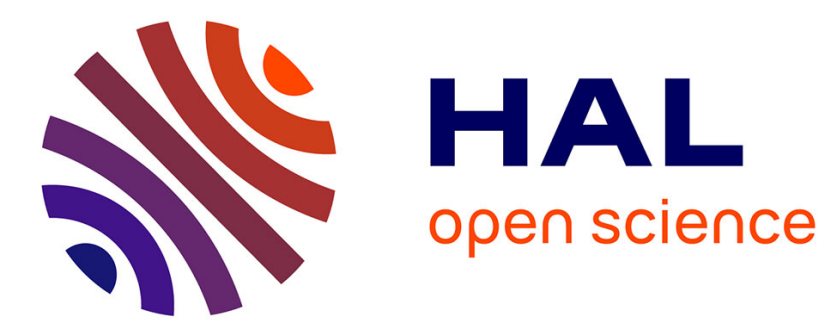

\title{
Macroscopic dynamics in nematics near the uniaxial-biaxial phase transition
}

\author{
H. Brand, J. Swift
}

\section{To cite this version:}

H. Brand, J. Swift. Macroscopic dynamics in nematics near the uniaxial-biaxial phase transition. Journal de Physique Lettres, 1983, 44 (9), pp.333-337. 10.1051/jphyslet:01983004409033300 . jpa00232200

\section{HAL Id: jpa-00232200 https://hal.science/jpa-00232200}

Submitted on 1 Jan 1983

HAL is a multi-disciplinary open access archive for the deposit and dissemination of scientific research documents, whether they are published or not. The documents may come from teaching and research institutions in France or abroad, or from public or private research centers.
L'archive ouverte pluridisciplinaire HAL, est destinée au dépôt et à la diffusion de documents scientifiques de niveau recherche, publiés ou non, émanant des établissements d'enseignement et de recherche français ou étrangers, des laboratoires publics ou privés. 
Classification

Physics Abstracts

$61.30 \mathrm{G}$

\title{
Macroscopic dynamics in nematics near the uniaxial-biaxial phase transition
}

\author{
H. Brand \\ Bell Telephone Laboratories, Murray Hill, New Jersey 07974, U.S.A. \\ and J. Swift \\ Dept. Physics, University of Texas, Austin, Texas 78712, U.S.A.
}

(Reçu le 15 décembre 1982, accepté le 17 mars 1983)

\begin{abstract}
Résumé. - Nous présentons une discussion des expériences possibles révélant les propriétés macroscopiques des nématiques uniaxes près de la transition uniaxe-biaxe. En particulier, nous proposons une mesure d'un nouveau couplage statique entre les gradients des fluctuations du directeur $(\delta n)$ de la phase uniaxe et du paramètre d'ordre $\left(\xi_{\mathrm{ij}}\right)$ de la phase biaxe.
\end{abstract}

\begin{abstract}
Based on the derivation of the macroscopic dynamics, we present a discussion of possible experiments which probe the macroscopic behaviour of uniaxial nematics close to the transition to the biaxial phase. In particular we propose an experiment to measure the influence of a novel static cross coupling between gradients of the director fluctuations $(\delta n)$ of the uniaxial phase and the order parameter $\left(\xi_{\mathrm{ij}}\right)$ of the biaxial phase.
\end{abstract}

Starting with the identification [1] of lyotropic biaxial nematics in 1980 there has been an increasing interest in the experimental $[2,3]$ and theoretical [Ref. 4 and references cited therein] investigation of the macroscopic properties of these systems. Very recently Jacobsen and one of the present authors [5] presented a hydrodynamic description of the uniaxial phase close to the transition to the biaxial phase. In addition to the strictly hydrodynamic variables of the uniaxial phase namely density $\rho$, entropy density $\sigma$, density of linear momentum $\mathbf{g}$ and the director fluctuations $\delta$ n (characterizing the spontaneously broken rotational symmetry) the authors of reference [5] introduced the order parameter $\xi_{i j}$ which is slow although with a long, but finite relaxation time $\tau$. The assumption which enters this description is, of course, that no additional « slow » quantities exist.

In the present note we generalize the statics given in [5] by including the effects of external magnetic fields. We also give a novel cross coupling term between the gradients of the director fluctuations and gradients of the quantity $\xi_{i j}$ (a symmetric, traceless tensor; cf. [5] for details). It seems important to notice that this cross coupling, unlike many of the other features discussed below, has no analogue near the uniaxial-isotropic phase transition. The latter has been studied in a series of papers by de Gennes [6].

To probe the new cross coupling experimentally we propose a static configuration in an 
inhomogeneous magnetic field. In addition we discuss which experiments can be done to study the static and dynamic behaviour in some detail; as it turns out there are strong similarities with the corresponding situations near the uniaxial-isotropic phase transition. It is important to note, however, one important difference between the two transitions. The isotropic-uniaxial transition is weakly first order (and associated with a latent heat) thus giving rise to finite fluctuations of considerable magnitude. The situation near the uniaxial-biaxial transition is, from an experimental point of view, even more favourable because this transition is, at least in mean field approximation [7] of second order, and the experimental results available so far seem to confirm these predictions. Thus, we can expect diverging fluctuations of various quantities when approaching the uniaxial-biaxial phase transition from above.

For the generalized Ginzburg Landau free energy we have

$$
\begin{array}{r}
F=\frac{1}{2} \int\left\{a \xi_{\mathrm{ij}} \xi_{\mathrm{ij}}+C_{\|}\left(n_{\mathbf{k}} \nabla_{\mathbf{k}} \xi_{\mathrm{ij}}\right)\left(n_{1} \nabla_{1} \xi_{\mathrm{ij}}\right)+C_{\perp} \delta_{\mathbf{k} \mathbf{1}}^{\mathrm{tr}}\left(\nabla_{\mathbf{k}} \xi_{\mathrm{ij}}\right)\left(\nabla_{1} \xi_{\mathrm{ij}}\right)+O\left(\xi^{4}\right)+\right. \\
\left.+\psi_{\mathrm{ijk} \mathbf{l m}}\left(\nabla_{\mathrm{i}} \xi_{\mathrm{jk}}\right)\left(\nabla_{1} n_{\mathrm{m}}\right)\right\} \mathrm{d}^{3} r+F_{0},
\end{array}
$$

where $\delta_{\mathrm{ij}}^{\mathrm{tr}}=\delta_{\mathrm{ij}}-n_{\mathrm{i}} n_{\mathrm{j}}$ and where the first two lines have been presented in reference [5]. $F_{0}$ contains the purely hydrodynamic contributions which can be found e.g., in reference [8]. The new cross coupling term has the detailed form

$$
\psi_{\mathrm{ijklm}}=\psi\left\{n_{\mathrm{i}} \delta_{\mathrm{j} 1}^{\mathrm{tr}} \delta_{\mathbf{k m}}^{\mathrm{tr}}+n_{\mathrm{i}} \delta_{\mathrm{jm}}^{\mathrm{tr}} \delta_{\mathbf{k} \mathbf{l}}^{\mathrm{tr}}+n_{1} \delta_{\mathrm{ij}}^{\mathrm{tr}} \delta_{\mathbf{k m}}^{\mathrm{tr}}+n_{1} \delta_{\mathrm{ik}}^{\mathrm{tr}} \delta_{\mathrm{jm}}^{\mathrm{tr}}\right\}
$$

The effects of an external magnetic field can be easily included into equation (1) giving rise to the additional terms $\left(F_{H}\right)$

$$
F_{H}=\int\left[\tilde{\chi}_{\mathrm{ijk} \mathbf{l}} H_{\mathrm{i}} H_{\mathrm{j}} \xi_{\mathbf{k} 1}+\tilde{\tilde{\chi}}_{\mathrm{ijk} \mathbf{m} \mathrm{n}} H_{\mathrm{i}} H_{\mathrm{j}} \xi_{\mathrm{k} \mathbf{1}} \xi_{\mathrm{mn}}\right] \mathrm{d}^{3} r
$$

where

$$
\tilde{\chi}_{i j k \mathbf{l}}=-\tilde{\chi}\left(\delta_{\mathbf{i l}}^{\mathrm{tr}} \delta_{\mathbf{j k}}^{\mathrm{tr}}+\delta_{\mathrm{ik}}^{\mathrm{tr}} \delta_{j \mathbf{j}}^{\mathrm{tr}}\right)
$$

and where $\tilde{\tilde{\chi}}$ contains two independent nontrivial terms giving rise to the contributions

$$
\tilde{\tilde{\chi}}_{1} H_{\perp}^{2} \xi_{\mathbf{k} 1}^{2}+\tilde{\tilde{\chi}}_{2} H_{\mathrm{i}} H_{\mathrm{j}} \xi_{\mathrm{il}} \xi_{\mathrm{j} 1} .
$$

where $H_{\perp}$ symbolizes the magnetic field perpendicular to $n$. It should be kept in mind, however, that $\tilde{\tilde{\chi}}$ contains an additional factor $\xi_{i j}$ compared to $\tilde{\chi}$ and is therefore presumably of minor importance. The dynamics remains unchanged, i.e., the reversible and the irreversible currents are a mere superposition of the terms given in [5] and [8]. No additional cross couplings appear.

A first simple application of the basic equation is magnetic birefringence in the plane perpendicular to the uniaxial director. If we assume a homeotropic sample with $\mathbf{n} / / \mathbf{z}$ (enforced e.g., by a static magnetic field in $z$-direction) we consider the effects of a static field in the $x y$-plane, say in the $x$-direction. We then find from equations (1) and (3) for the $x y$-plane

$$
\left\langle\xi_{\mathrm{ij}}\right\rangle=\frac{2 \tilde{\chi}}{a}\left[H_{\perp \mathrm{i}} H_{\perp \mathrm{j}}-\frac{H_{\perp}^{2}}{2} \delta_{\mathrm{ij}}^{\mathrm{tr}}\right],
$$

and especially in the $x$-direction

$$
\left\langle\xi_{x x}-\xi_{y y}\right\rangle=\frac{2 \tilde{\chi}}{a} H_{x}^{2}
$$


Proceeding in the same way as in [6] we find for the anisotropy of the refractive indices in the plane

$$
n_{\|}-n_{\perp}=\alpha H_{\perp}^{2}
$$

where $/ /$ and $\perp$ refer to the directions parallel and perpendicular to the magnetic field in the plane. Since $\alpha \sim 1 / a$ the effects predicted by equation (7) will become very large near the phase transition (cf. also Ref. [2] for a discussion of this point). Of course, an analogous argument goes through for electric birefringence in the $x y$-plane. Next we consider the case of flow birefringence. We assume as above that the uniaxial director is oriented in the $z$-direction, furthermore we consider a flow velocity along $x$ with the velocity gradient $\partial v_{x} / \partial y$.

We then get from the basic equations (Eq. (1) and Eqs. (6), (9), (12), (16)-(24) of ref. [5])

$$
\begin{aligned}
& \phi_{i j}=\tau_{i j k 1} A_{k 1}+\zeta_{i j k 1} \chi_{0}^{-1} \xi_{k 1}, \\
& \dot{\xi}_{i j}=-\phi_{i j},
\end{aligned}
$$

where we use the notation of reference [5] where possible (e.g., $\left.A_{\mathrm{ij}}=\frac{1}{2}\left(\nabla_{\mathrm{i}} V_{\mathrm{j}}+\nabla_{\mathrm{j}} V_{\mathrm{i}}\right)\right)$.

For the configuration we have chosen, we assume a time dependence of the form $\mathrm{e}^{i \omega t}$ and obtain

$$
\xi_{x y}=-\frac{\tau A_{x y}}{i \omega+\zeta \chi_{0}^{-1}}
$$

In deriving (9) we have kept the viscous term $\zeta$ and the bending energy of the $\xi$-field. It is well known for the isotropic-uniaxial nematic transition (9) that the effects of the bending energy can be neglected. Then we deduce from equation (9) in the low frequency limit

$$
\xi_{x y}(\mathbf{k})=-\frac{\tau}{a \zeta} A_{x y}(\mathbf{k})
$$

Proceeding as in the uniaxial-isotropic case [0] from equation (10) we get for the difference in the refractive indices

$$
n_{\|}-n_{\perp}=\tilde{\alpha} \tilde{\tau} \frac{\partial v_{x}}{\partial y}
$$

where the characteristic time $\tilde{\tau}$ diverges at the transition; for the constant $\tilde{\alpha}$ we refer to [6]. From the basic equations it is easily checked that all other variables $\left(\mathrm{e} . \mathrm{g} . \delta n_{\mathrm{i}}\right)$ do not couple in the specific configuration we have chosen. As soon as the equilibrium value of the uniaxial director $\mathbf{n}_{\mathbf{0}}$ has a component in the plane of the shear, however, the above analysis has to be changed completely and no simple result probing the magnitude of $\xi_{\mathrm{ij}}$ can be obtained.

The influence of fluctuations of $\xi_{\mathrm{ij}}$ can also be investigated by looking at the shear wave attenuation. We assume, as above, $\mathbf{n}_{0} / / \hat{z}$ and the velocity in $x$-direction with variations in the $y$-direction. Then from equations (12),..., (26) of [5] we can extract for the stress tensor

$$
\sigma_{x y}=-2 v_{2} A_{x y},
$$

and for the dynamic equations of the order parameter

$$
\dot{\xi}_{x y}=-\tau A_{x y}-\xi \chi_{0}^{-1} \xi_{x y} .
$$


Combining equations (12) and (13) we get, after a Fourier transform, the effective viscosity $\eta_{\text {eff }}$

$$
\eta_{\text {eff }}(\omega)=v_{2}+\frac{1}{2} \frac{\chi_{0}^{-1} \tau^{2}}{i \omega+\zeta \chi_{0}^{-1}} .
$$

Thus we get a finite correction for $\eta_{\text {eff }}(\omega=0)$ of the order $\tau^{2} / \zeta$ which gives a possibility to measure this quantity because at higher frequencies $\left(\omega^{2} \gg\left(\zeta \chi_{0}^{-1}\right)^{2}\right)$ there is no correction. We mention in passing that our expression for the shear viscosity differs significantly from the result derived by de Gennes for the uniaxial-isotropic phase transition. De Gennes finds, for example, no static correction. Furthermore, there is a high frequency correction in the latter phase transition [6].

Finally, we discuss the possibilities of measuring the static cross coupling presented here for the first time. Light scattering in simple geometries has been discussed in reference [5]. As is easily checked, the coefficient $\psi$ does not enter for these special cases, thus leaving the presentation of [5] unchanged. It is straightforward to convince oneself, however, by looking into more complicated configurations, that $\psi$ enters in general situations, although the dependence on $\psi$ becomes rather involved. In the present paper we propose a static experiment to gain some qualitative insight into the effects of $\psi$. To achieve this one sees, by inspection of equation (1), that one needs inhomogeneous external fields, and a magnetic field might be a particularly good candidate. We examine the situation sketched in figure 1 . We assume that one pole of the magnet is wedge shaped in the $x y$-plane and extends to $\pm \infty$ in $y$-direction which renders the situation essentially two-dimensional. In the uniaxial phase the $\mathbf{n}$ field is then distorted, but in a symmetric way in $z$ and $-z$-direction because the twist term $K_{2}$ does not enter. If the uniaxial phase is cooled towards the biaxial phase transition the $\psi$-term, which breaks the $z \rightarrow-z$ symmetry in the configuration chosen here, becomes more and more important and thus the pattern (observed e.g., in a microscope) picks up an asymmetric distortion (in z-direction), which should be detectable experimentally. This can be inferred from the variational equations obtained from (1), (3)

$$
\frac{\delta F}{\delta \xi_{\mathrm{ij}}}=0 ; \quad \frac{\delta F}{\delta n_{\mathrm{i}}}=0
$$

In conclusion we have discussed possible experimental configurations to study the influence of the order parameter near the uniaxial-biaxial nematic phase transition. In particular we have proposed an experimental set up to study the novel coupling term between the director field

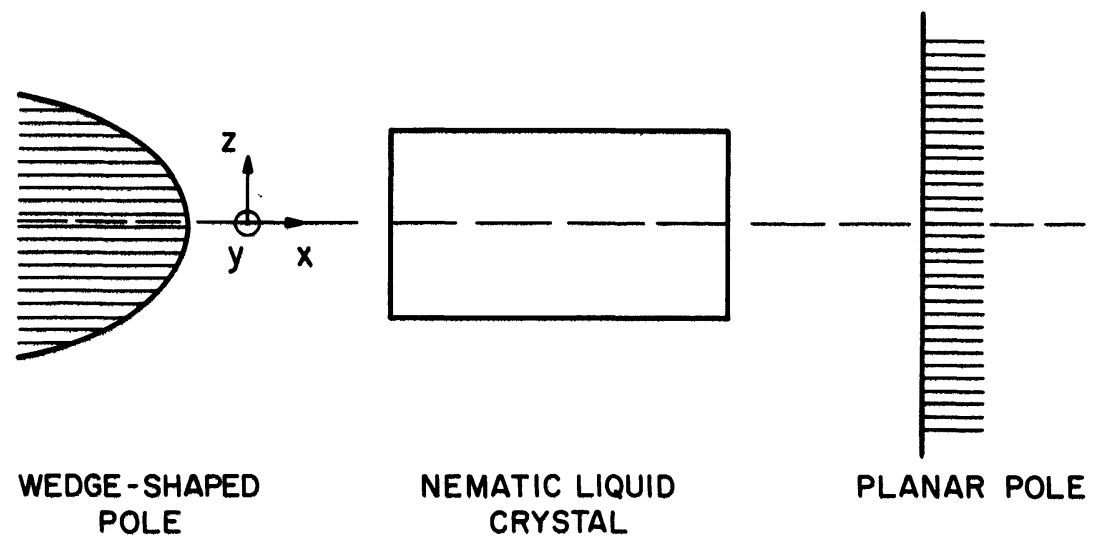

Fig. 1. - Proposed experimental set up to detect the cross-coupling coefficient $\psi$ by application of an inhomogeneous magnetic field. Without the inhomogeneous field one can assume a homogeneous texture, if necessary this can be enforced by a homogeneous field in $z$-direction. 
and the slowly varying quantity $\xi_{\mathrm{ij}}$ which should allow for a simple qualitative determination of the importance of this term.

\section{Acknowledgments.}

It is a pleasure to thank Pat Cladis, W. D. McCormick and Ron Pindak for useful discussions. The research of one of us (J.S.) was supported in part by the Robert A. Welch Foundation under grant number F-767.

\section{References}

[1] YU, L. J. and SAuPe, A., Phys. Rev. Lett. 45 (1980) 1000.

[2] SaUPe, A., Boonbrahm, P. and Yu, L. J., preprint.

[3] Bartolino, R., Chiaranza, T., Meuti, M. and Compagnoni, R., Phys. Rev. A 26 (1982) 1116.

[4] Brand, H. and Pletner, H., Phys. Rev. A 24 (1981) 2777, Phys. Rev. A 26 (1982) 1783.

[5] JACOBSEn, E. A. and Swift, J., Mol. Cryst. Liq. Cryst. 87 (1982) 29.

[6] De Gennes, P. G., Phys. Lett. A 30 (1969) 454, Mol. Cryst. Liq. Cryst. 12 (1971) 193, Physics of Liquid Crystals (Clarendon Press, Oxford) 1974; for a review of the experiments near the uniaxialisotropic phase transition we refer to de Ǵennes' book p. 211-215 and to ChandRasekHAR, S., Liquid Crystals, p. 64-74.

[7] Freiser, M. J., Phys. Rev. Lett. 24 (1970) 1081 ;

AlBen, R., Phys. Rev. Lett. 30 (1973) 778.

[8] Forster, D., Hydrodynamic Fluctuations, Broken Symmetry and Correlation Functions (Benjamin, Reading) 1975. 